\title{
Application and Implementation of Traffic Violation Report Combined with Vision and Voice Kits
}

\author{
Wen-Chuan $\mathrm{Wu},{ }^{1 *}$ Yen-Lun Chen, ${ }^{2}$ and Pei-Yu Lin ${ }^{3 * *}$ \\ ${ }^{1}$ Department of Computer Science and Information Engineering, National Ilan University, \\ No. 1, Sec. 1, Shennong Rd., Yilan City, Yilan County 260, Taiwan, R.O.C. \\ ${ }^{2}$ Department of Computer Science and Information Engineering, Aletheia University, \\ No. 32, Zhen-Li St., Tamsui Dist., New Taipei City 25103, Taiwan, R.O.C. \\ ${ }^{3}$ Department of Information Communication, Innovation Center for Big Data and Digital Convergence, \\ Yuan Ze University, No. 135, Yuan-Tung Rd., Chung-Li 32003, Taiwan, R.O.C.
}

(Received July 6, 2021; accepted November 25, 2021; online published December 14, 2021)

Keywords: Internet of Things, sensor, vision kit, voice kit, vehicle violation report, cloud storage

The Internet of Things (IoT) is a technique to connect various devices together in a network and exchange data among themselves. Currently, there are many IoT applications, such as smart homes, smart cities, smart manufacturing, and so forth. Their purpose is to make life more convenient. In this paper, we use IoT technology to solve the problem of traffic violation reports. We utilize specific sensors and a signal processing technique to detect events or changes in the environment. Then, messages are sent to trigger a camera to obtain videos of the violation. These video clips and the related information are finally uploaded to the cloud space of the reporter of the violation. Experimental results showed that the proposed scheme can speed up traffic violation reporting and can increase the willingness of people to act as whistleblowers.

\section{Introduction}

Transportation in Taiwan is well developed and convenient. To reach tourist spots or other cities, people can take public transportation, such as Mass Rapid Transit (MRT), buses, and taxis, or use motorcycles and cars to move themselves quickly. However, private vehicles are still generally used in transportation. According to statistics, ${ }^{(1)}$ the highest density of motorcycles in the world is in Taiwan, with almost one per person. The main reason is their high mobility and that they are less affected by traffic jams than other vehicles, so motorcycles have become the primary transportation tool for most commuters. In addition, the number of private cars in Taiwan has increased significantly in recent years, with an increase of 160000 vehicles from 2016 to $2018 .^{(2)}$ Nearly $60 \%$ of families own a private car and nearly $30 \%$ have two private cars. On average, there are 1.5 private cars per family.

The rapid increase in the numbers of automobiles and motorcycles in Taiwan has resulted in many traffic violations, such as illegal parking, running of red/yellow lights, wrong-way driving, lane changes without signaling, and speeding. These may lead to traffic accidents and even

\footnotetext{
*Corresponding author: e-mail: wenn@,niu.edu.tw

**Corresponding author: pagelin3@gmail.com

https://doi.org/10.18494/SAM.2021.3506
} 
deaths. Based on statistics ${ }^{(3)}$ from New Taipei City, the incidence of traffic accidents and related mortality in 2019 increased by 32.23 and 33.06\%, respectively, compared with the previous year.

To maintain road safety and to reduce traffic accidents, the police in many cities of Taiwan have recently clamped down on driving violations and illegal parking on roads that are prone to congestion and accidents. People can also film evidence of traffic violations through tools such as smartphones and dash cams, ${ }^{(5)}$ in addition to the police. Table 1 shows the statistics of violations reported by Taipei City from 2015 to 2019. More than half of the violations are by cars. Table 2 shows the statistics of reported violations for New Taipei City. It can be seen that the top three reports of violations in the past five years are for illegal parking, speeding, and running red lights. Nowadays, people have digital tools that can be used to film traffic violations, but the process required to edit evidence of violations is time-consuming and labor-intensive. If digital technology can be used to solve this problem, it will increase people's willingness to report violations, helping to reduce their number and reduce the burden on the traffic police.

In the past, evidence of a violation had to clearly include a recording of the license plate, location, and status of the offending vehicle, which was then uploaded to the reporting website responsible for the jurisdiction. It takes a long time to tidy up digital evidence and violation information. There are now apps to make it easier for whistleblowers to submit violation information, such as InMyEye ${ }^{(7)}$ (a Line reporting bot), the Police Report app, ${ }^{(8)}$ and FixTW. ${ }^{(9)}$ People can manually fill in their personal information and upload the content and evidence of the violation. However, these three apps are more suitable for pedestrians and other non-drivers than for drivers. When a reporter is driving a motorcycle or a car, the app cannot be used at the same time, and it is necessary to edit lengthy video taken by the dash cam afterwards, which is timeconsuming and laborious. ${ }^{(10,11)}$ There have been various attempts to automate video editing, such as applying a user-specified combination of film-editing idioms, ${ }^{(12)}$ audio-visual continuity, ${ }^{(13)}$ and so forth. To speed up this editing, in this paper, we propose the use of Internet of Things (IoT) technology ${ }^{(14-17)}$ to make it easier for drivers to report violations.

Table 1

Traffic violations in Taipei City. ${ }^{(4)}$

\begin{tabular}{lccc}
\hline Year & Number of reports & Percentage by cars (\%) & Percentage by motorcycles (\%) \\
\hline 2015 & 1802705 & 55.10 & 41.98 \\
\hline 2016 & 1917990 & 57.52 & 39.92 \\
\hline 2017 & 1909152 & 56.69 & 40.82 \\
\hline 2018 & 2131596 & 56.34 & 41.24 \\
\hline 2019 & 2382422 & 56.91 & 40.53 \\
\hline
\end{tabular}

Table 2

Traffic violations in New Taipei City. ${ }^{(6)}$

\begin{tabular}{lcccc}
\hline Year & Number of reports & First & Second & Third \\
\hline 2015 & 1245925 & Illegal parking 44.09\% & Speeding 15.35\% & Running red lights 12.83\% \\
\hline 2016 & 1683292 & Illegal parking $53.31 \%$ & Speeding 11.71\% & Running red lights 10.67\% \\
\hline 2017 & 1657944 & Illegal parking 48.54\% & Speeding 12.52\% & Running red lights $11.35 \%$ \\
\hline 2018 & 1934814 & Illegal parking 47.36\% & Speeding 13.04\% & Running red lights $10.80 \%$ \\
\hline 2019 & 2161140 & Illegal parking 46.06\% & Running red lights 11.51\% & Illegal U-turns 11.39\% \\
\hline
\end{tabular}




\section{Traditional Traffic Violation Report Process}

At present, police agencies throughout Taiwan have traffic violation reporting websites. If a violation is found, people can gather evidence of the violation and submit an online report to the relevant police jurisdiction within seven days from the date of the violation. Figure 1 is a flow chart for reporting traffic violations. First, it is necessary to sign in to a reporting website, and then fill in the personal information of the reporter, such as the name, ID number, e-mail, contact mobile number, and contact address. The reporter will receive a certification letter in their mailbox, and then the content of the report can be entered online after replying to the certification letter. Once the $30 \mathrm{~min}$ certification time has passed, the reporter will be authenticated again to allow them to report another violation. Figure 2 shows the website ${ }^{(18)}$ for reporting traffic violations in New Taipei City, where the upper part is the report content and the lower part is the reporter information.

The evidence provided by the reporter must be complete and include video clips or multiple consecutive photos, and the license plate and ground markings must be clearly identifiable. The date, time, and location of the violation must also be included. Figure 3 shows four examples of traffic violations: illegal parking, running a red light, illegal overtaking, and riding without a helmet. Only a single photograph is required to report a rider without a helmet, but in other cases, it is recommended that at least three consecutive photos or video clips are provided. In the case of a "lane change without signaling", it is impossible to determine whether the driver used the turn signal only from continuous and static photos. For this reason, it is recommended that videos are submitted as evidence to increase the success rate of reporting.

However, the volume of video data is very large. A 128 GB memory can store almost 450 videos of 1080p quality with a total time of $22 \mathrm{~h}$. Moreover, if a video is edited manually, it will take $3-5 \mathrm{~min}$ to edit a $3 \mathrm{~min}$ file on average. Although some dash cams now have a built-in WiFi function, meaning that people can use a mobile phone to edit videos by connecting it to the dash cam, the time required to edit videos is still high.

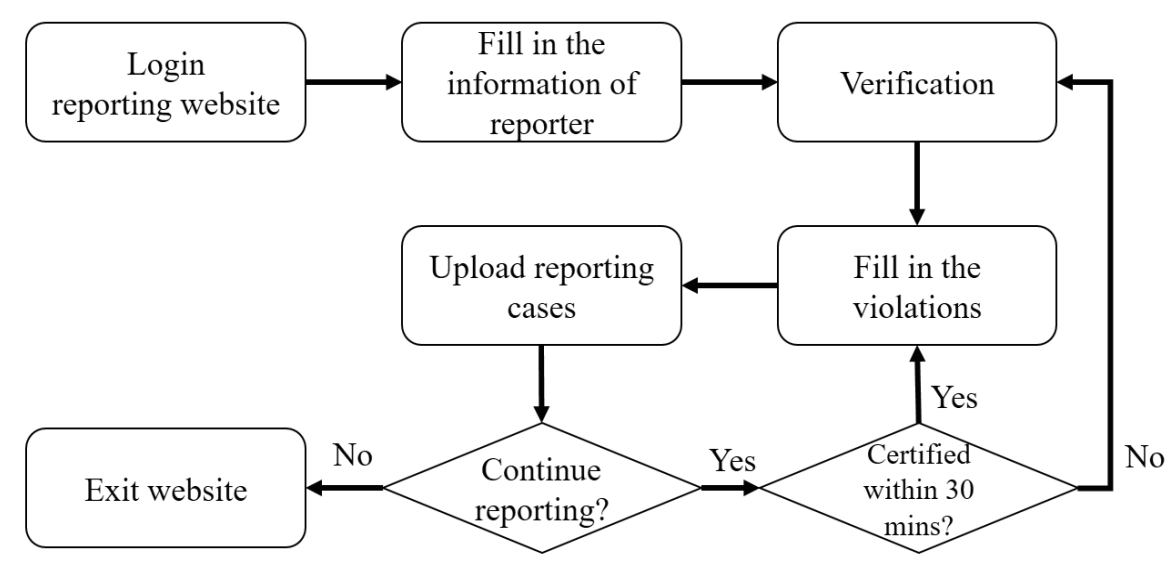

Fig. 1. Flowchart to report traffic violations. 

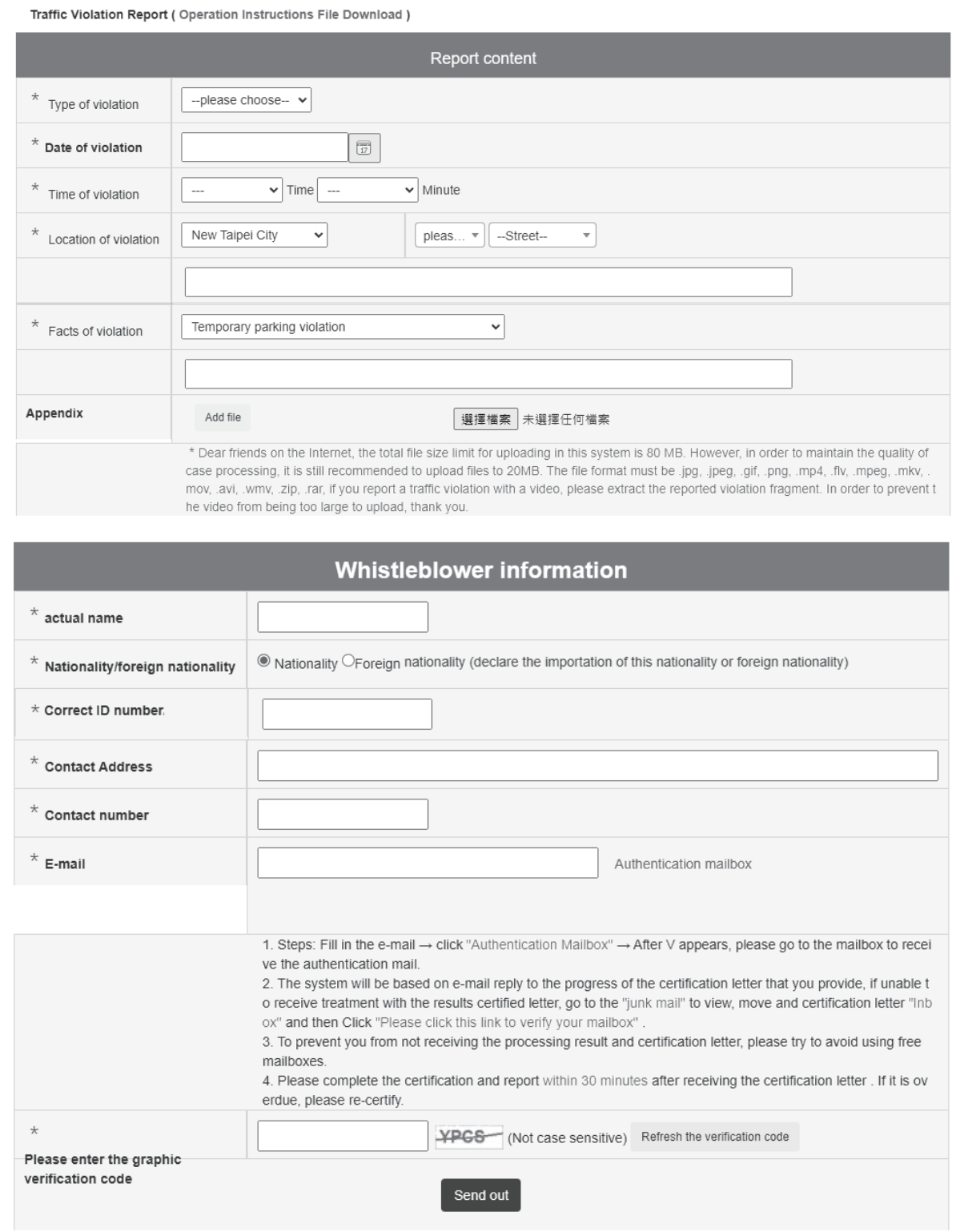

Fig. 2. New Taipei City's website for reporting traffic violations.

\section{Proposed Scheme}

To speed up the video editing work of traffic reporting, the proposed scheme adopts multiple IoT modules so that violation clips can be automatically stored while driving. Figure 4 shows the hardware system architecture in the proposed scheme. It is designed to use a single-clip microcomputer, Raspberry Pi, mainly because of its small size and low power consumption. In general, a driver will be focused on the traffic conditions in front of the windshield while driving and may also observe many vehicle violations. Therefore, in this work, an input button is connected to the Raspberry Pi. If the driver detects a violation of vehicles, they can press the button. In addition, instructions can be given to the Raspberry Pi via a voice module. At this 


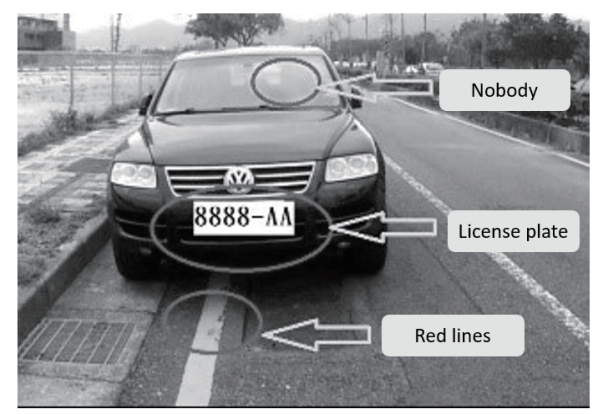

(a)

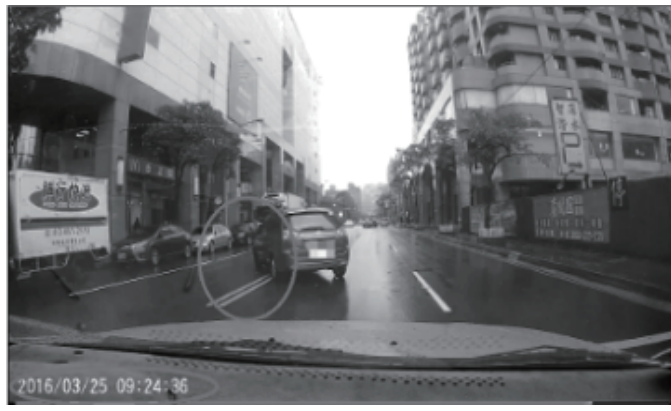

(c)

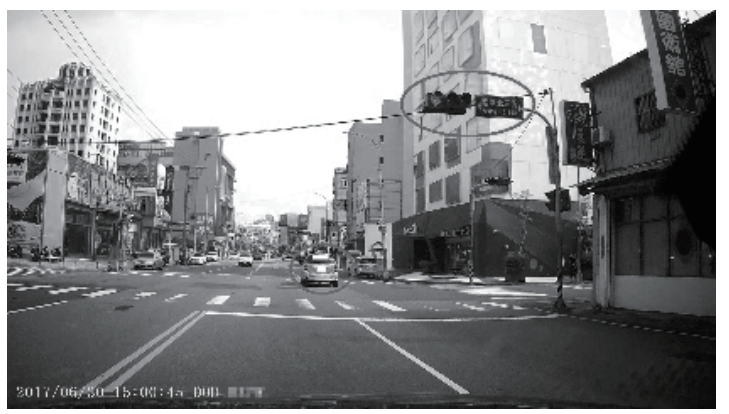

(b)

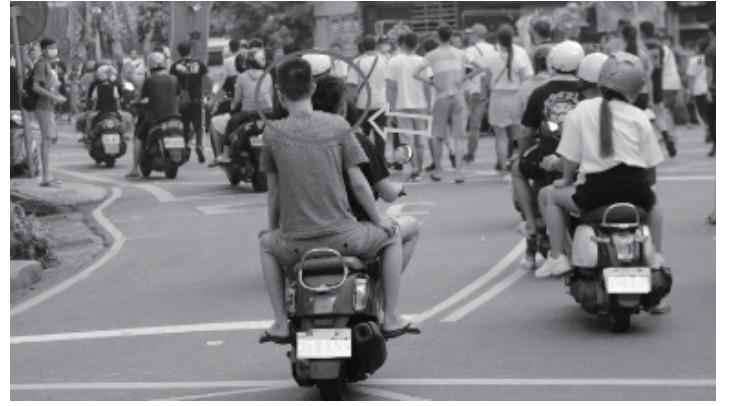

(d)

Fig. 3. Examples of traffic violations. (a) Illegal parking, (b) running red light, (c) illegal overtaking, and (d) riding without a helmet.

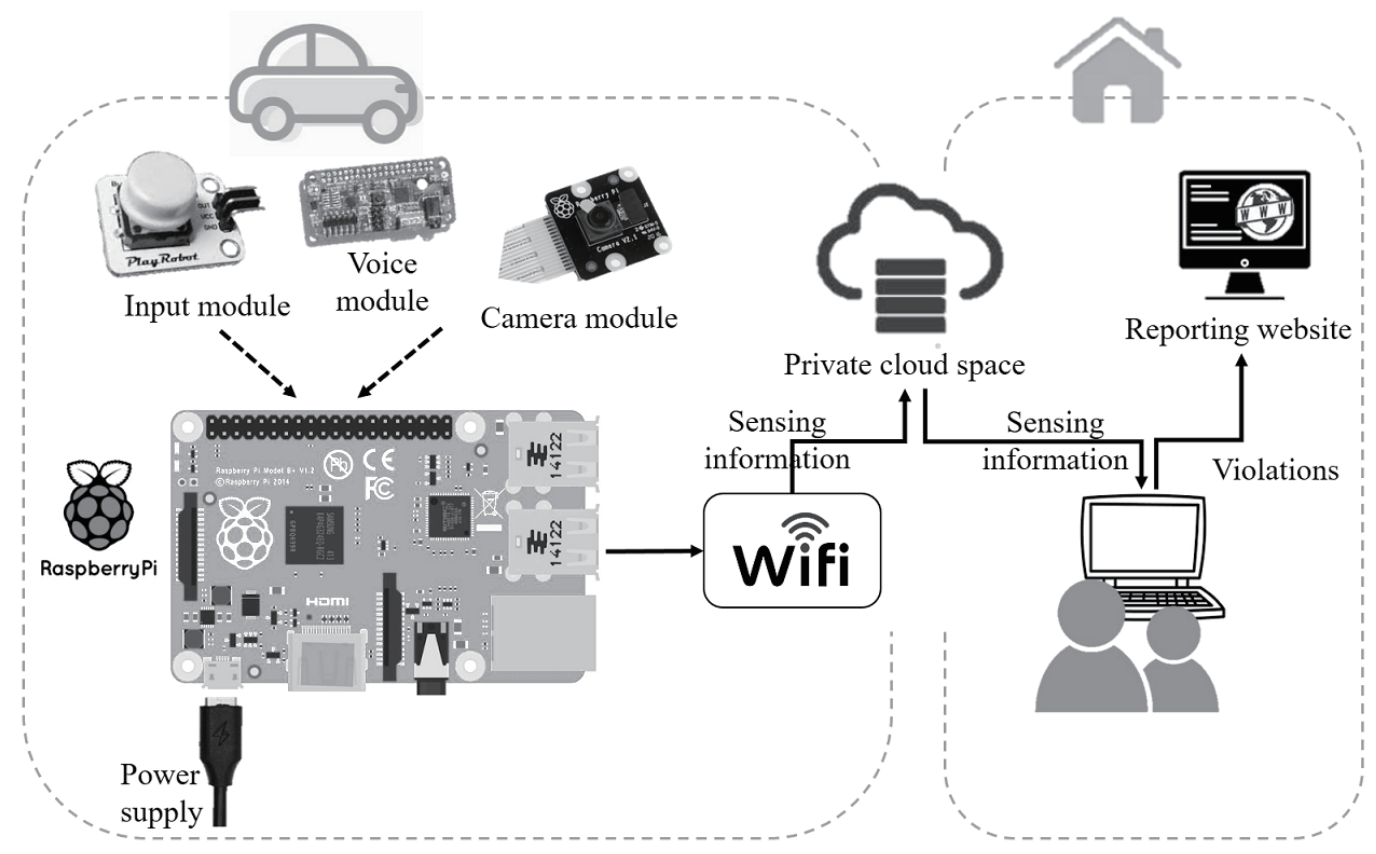

Fig. 4. Architecture of the proposed scheme.

time, the Raspberry Pi will trigger the connected camera to record a video. Here, the length of a video clip in the proposed scheme is $10 \mathrm{~s}$. The file size of the $10 \mathrm{~s}$ video in MP4 format is about 
$5 \mathrm{MB}$, which does not exceed the upper limit of 10-100 MB set by each city police department.

The input module of this system is recommended to be placed near the steering wheel, allowing the driver to operate it quickly. This will not affect driving safety or distract the driver. In addition, the video clips must be marked with the date and time of the violation for legal requirements. Therefore, the "annotate_text" attribute of the picamera library is used in the codes of our system to convert the date and time into text and superimpose it on the picture captured by the camera. Our system uses the Rclone technique ${ }^{(19,20)}$ to mount the cloud service of Google Drive, and these video clips are uploaded to a private cloud space synchronously through the Wi-Fi wireless network of the Raspberry Pi. This enables the whistleblower to download the video materials after returning home, and upload them to the reporting website together with their personal information. The following are the operating steps for the Rclone open source software connected to the Google Drive cloud space service:

Step 1: Install Rclone software in Raspberry Pi.

Step 2: Adjust the Rclone config settings, provide the mount name, and set the client ID.

Step 3: Obtain the API key certification of a specific Google Drive and enable it.

Step 4: Write the "rclone sync" command in the script file (*.sh) to synchronize the files in the source directory and the destination directory.

Step 5: Run the script file (*.sh) in the Python program.

The operation of the voice module in our system is built on the snowboy website, which is used to train our own hotwords. The operation process of a smart speaker has four steps. First, a customized wakeword is created to wake up the device. Second, speech-to-text (STT) software is used to convert user speech into text, where we use the speech recognition API of Google. Next, there is a text understanding step to understand the natural language of the user. Here, we use a natural language processing (NLP) tool named Dialogflow provided by Google to understand what the user is saying. Dialogflow is used to build an agent AI to distinguish entity and intent in the sentences. For example, when a user says "How's the weather tomorrow?" or "Will it rain in Taipei next week?", the entity is the date/location and the intent is the weather. Then, we add a training phrase to a Dialogflow intent, which contains the word "Recording". Dialogflow will automatically recognize it and trigger the entity. Finally, text-to-speech (TTS) software is used to convert the understood text into speech and output it. Here, we use the speech recognition API of Google to implement STT as well as TTS.

\section{Experimental Results}

In experiments, we use a Google AIY Voice Kit V1.0 to simulate the proposed scheme and connect it to a Raspberry Pi 4 and a Camera Module v2. This voice kit contains an arcade-style push button, a Voice HAT accessory board, a microphone board, and a 3-inch speaker. The Voice HAT accessory board is plugged into the 40 pins of the Raspberry Pi. Here, we use the Raspberry Pi 4 Model B, which has a built-in Wi-Fi wireless network. In addition, we also installed a GPS module and a Raspberry Pi Camera Module v2, where the former is used to record the GPS location and the latter has a high-quality image sensor with 8 megapixels, which supports 1080p 30 and 720p 60 videos. The proposed scheme is developed in Python language, 
and the camera resolution is set to $1280 \times 720 \mathrm{p}$ in the codes. Figure 5 shows the system implemented for the proposed scheme. There is a push button for input on the top as shown in Fig. 5(a), with a camera in front of the button as shown in Fig. 5(b). That AIY kit in a box is connected to the Raspberry Pi, Voice HAT board, and speaker, as shown in Fig. 6. It is necessary to connect the system to the Internet and power it with a portable charger, as shown in Figs. 5(c) and 5(d), to start the Raspberry Pi.

We performed an experiment in which we drove a car around Tamsui in New Taipei City for an hour. Our route passed through downtown streets and alleys. Figure 7 shows traffic video clips recorded using the proposed scheme, which are stored in a private cloud space. Static pictures taken by the proposed scheme and the original dash cam are shown in Figs. 8 and 9,

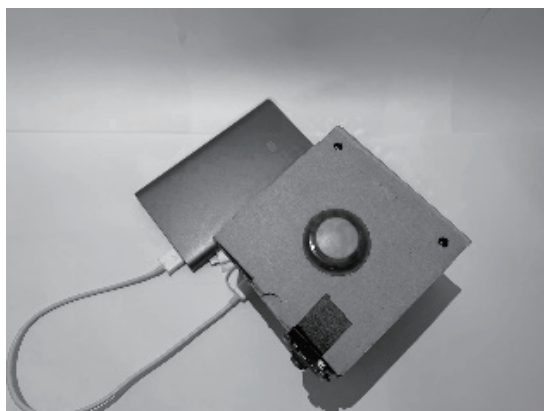

(a)

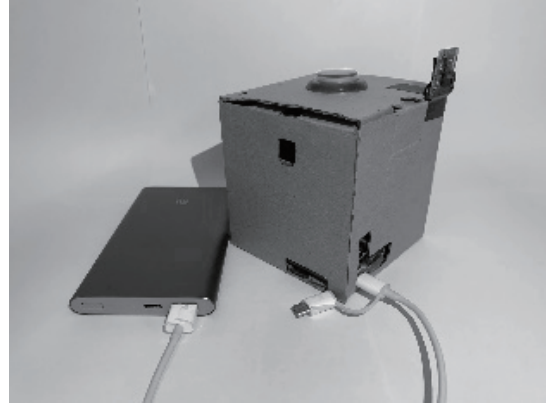

(c)

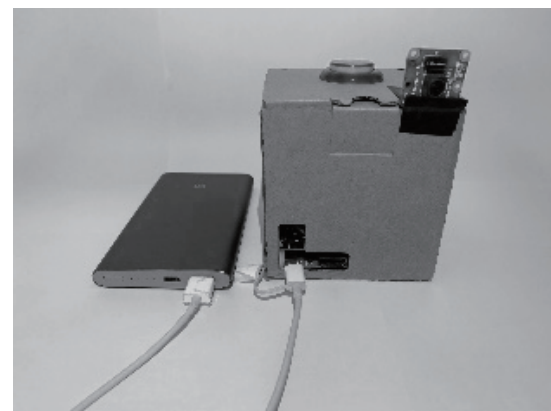

(b)

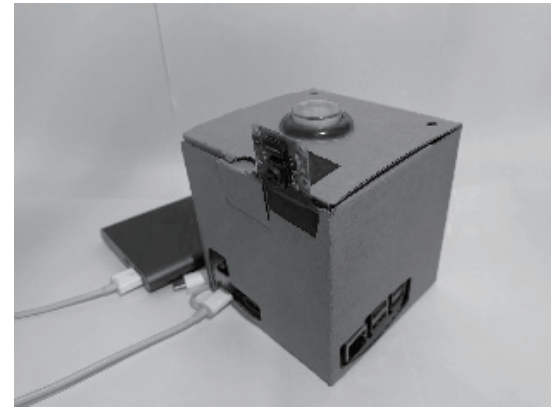

(d)

Fig. 5. Implemented system. (a) Top view, (b) front view, (c) left view, and (d) right view.
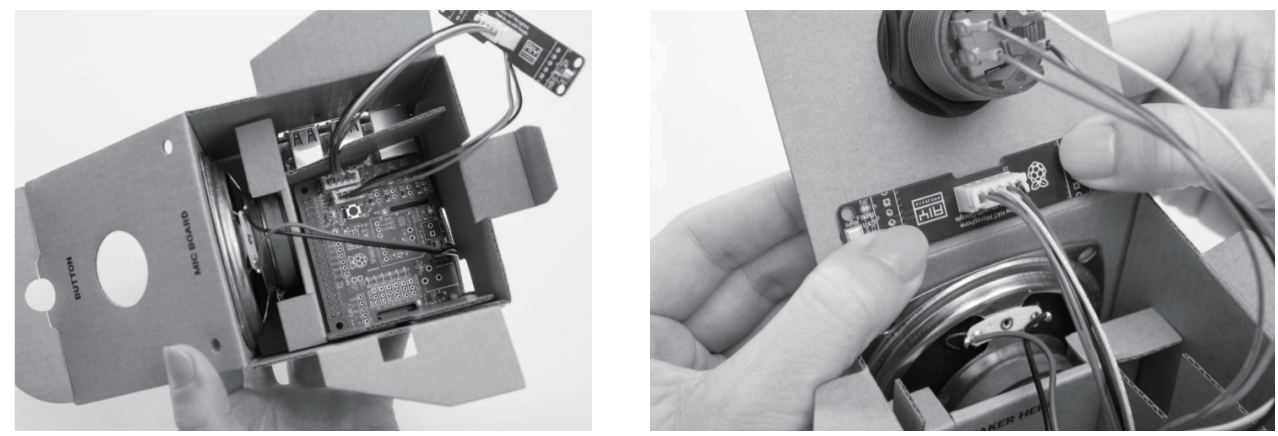

Fig. 6. Process of device installation. 


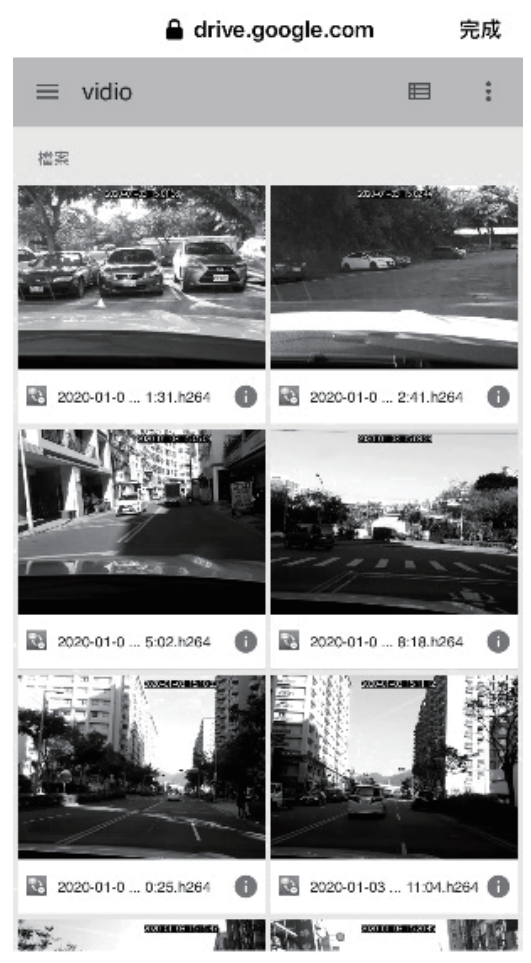

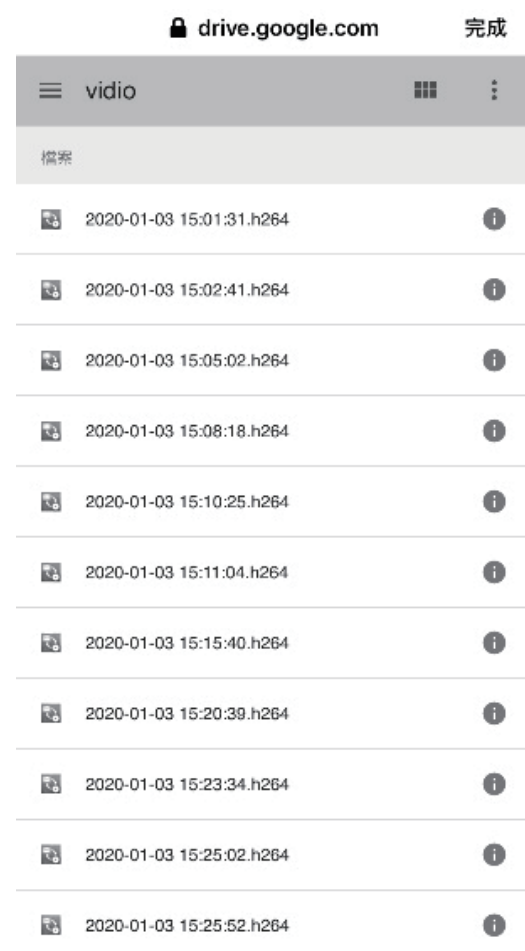

Fig. 7. Video data in private cloud space.

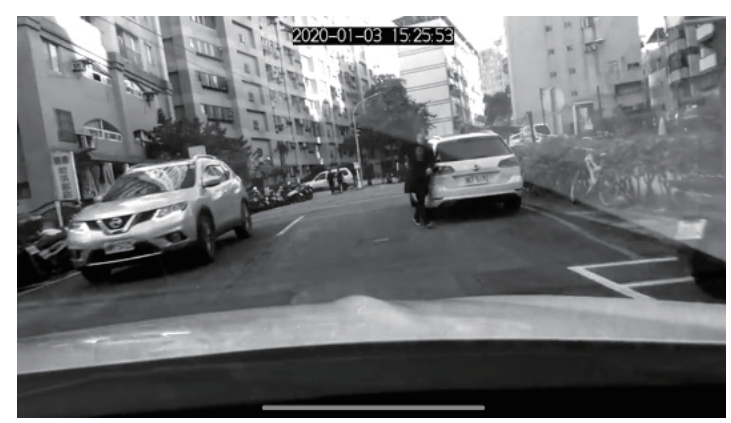

(a)

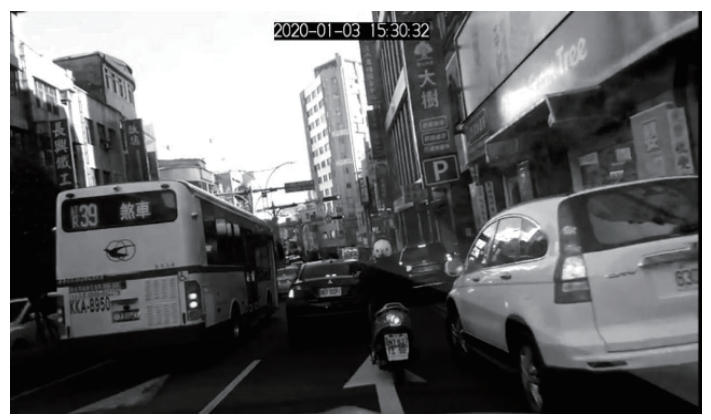

(b)

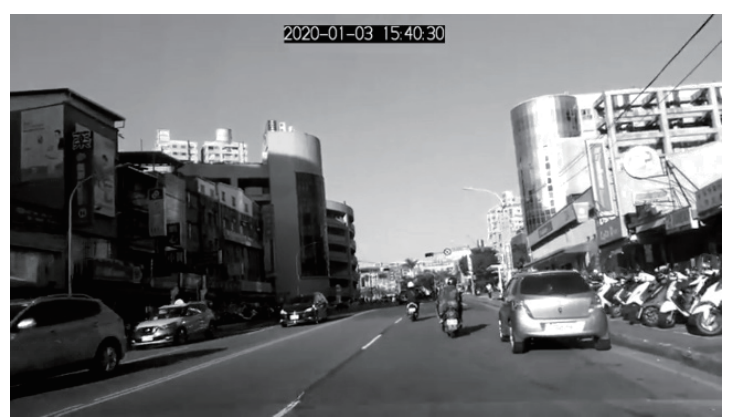

(c)

Fig. 8. Static pictures taken by the proposed scheme. (a) Beixin Road, (b) Zhongshan Road, and (c) Zhongshan N. Road. 


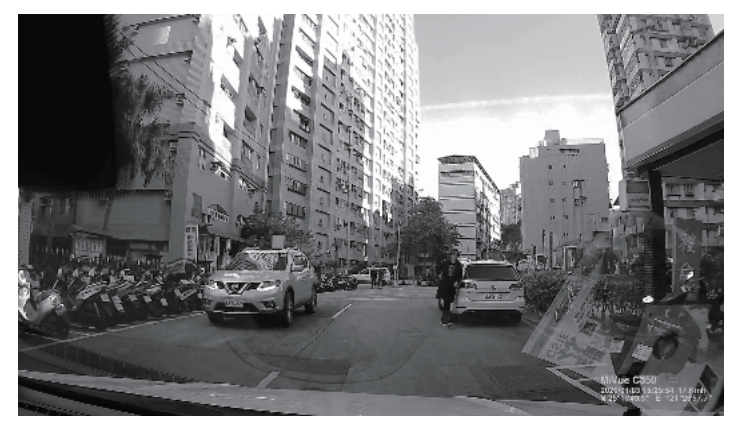

(a)

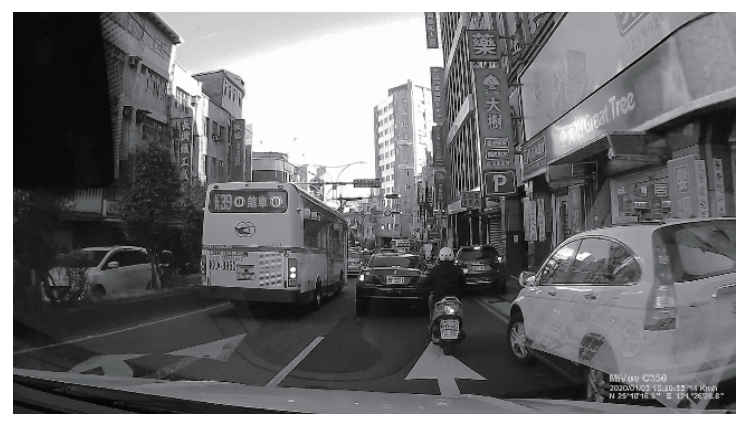

(b)

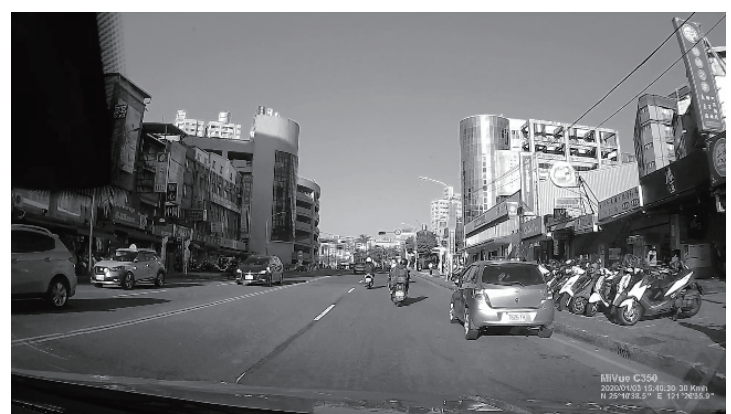

(c)

Fig. 9. Static pictures taken by the dash cam. (a) Beixin Road, (b) Zhongshan Road, and (c) Zhongshan N. Road.

Table 3

Success rate of voice instruction test.

\begin{tabular}{lcccc}
\hline Voice instruction & $\begin{array}{c}\text { Number of } \\
\text { successes }\end{array}$ & $\begin{array}{c}\text { Total number of } \\
\text { tests }\end{array}$ & $\begin{array}{c}\text { Success rate } \\
(\%)\end{array}$ & $\begin{array}{c}\text { Failure rate } \\
(\%)\end{array}$ \\
\hline Snowboy & 46 & 50 & 92 & 8 \\
\hline Start recording (in Chinese) & 42 & 50 & 84 & 16 \\
\hline Start recording (in English) & 41 & 50 & 82 & 18 \\
\hline Record & 38 & 50 & 76 & 24 \\
\hline
\end{tabular}

respectively. Both of them indicate the shooting date and time on the pictures. A Mio C350 dash cam was used in the car, whose resolution is as high as $1920 \times 1080$ p. Hence, the images in Fig. 9 have higher quality than those in Fig. $8(1280 \times 720$ p). By comparing Figs. $8(a)$ and 9 (a), it can be seen that the license plate captured at the same time in both images is clear and identifiable. Figure 8(b) shows that a $10 \mathrm{~s}$ clip recorded using the proposed scheme is able to record the violation of two adjacent cars parked illegally. Note that in strong sunlight, the license plate captured by our scheme is too bright to be recognized as shown in Figs. 8(c) and 9(c). This problem will be tackled in the future. Table 3 shows the success rate of a voice instruction test using our system. Here, we gave four voice instructions, where "snowboy" was used as the wakeword to wake up the AIY Voice device and the other devices. It can be seen from the table that the success rate of the wakeword is $92 \%$. It is also clear that the longer the customized voice instruction, the higher the success rate. 


\section{Conclusions}

The reporting of traffic violations needs to be supported by digital evidence. Most of such evidence, including consecutive photos or video clips, is edited from dash cams of cars, which is time-consuming and laborious. In this paper, IoT technology is used to connect multiple modules to communicate with each other. A driver discovering a violation can operate the input module or voice module and then the camera is activated to record a video clip. These videos and related information will be automatically uploaded to a specific private cloud space, which can reduce the time required for a whistleblower to report traffic violations. We found by simulation that the edited clips can be successfully used on a violation reporting website, and a whistleblower does not have to spend time editing videos in the dash cam, approximately halving the report processing time.

\section{References}

1 Agencia EFE: Taipei's Motorbike Waterfall a Sample of Asia's Most Popular Vehicle (2018). https://www.efe. com/efe/english/life/taipei-s-motorbike-waterfall-a-sample-of-asia-most-popular-vehicle/50000263-3673590

2 Ministry of Transportation and Communications: Investigation Report on the Use of Private Cars (2019). https://www.motc.gov.tw/ch/home.jsp?id=56\&parentpath=0,6 (accessed June 2021).

3 New Taipei City Police Department: Overview of A1-type Traffic Accidents in 2019 of New Taipei City (2020). https://www.police.ntpc.gov.tw/dl-17650-651e8clb-a666-40f5-99aa-12f271254d01.html

4 Traffic Division of Taipei City Police Department: Statistics on traffic violations of Taipei City over the years (2020). https://td.police.gov.taipei/Default.aspx

5 A. Matsuda, T. Matsui, Y. Matsuda, H. Suwa, and K. Yasumoto: Sens. Mater. 33 (2021) 17. https://doi. org/10.18494/SAM.2021.2998

6 New Taipei City Police Department: Statistics bulletin in New Taipei City Police https://www.police.ntpc.gov. tw/lp-2358-1.html (accessed June 2021).

7 InMyEye (Line reporting bot): https://ine.me/R/ti/p/\%40791kveqr (accessed May 2021).

8 Police Report APP: https://www.youstar.com.tw/?page id=2504 (accessed June 2021).

9 FixTW: Automated Traffic Violation Reporting Service https://fixtw.com/ (accessed May 2021).

10 Z. Wang: Proc. Int. Conf. Computers, Information Processing and Advanced Education (2021) 1446-1449. https://doi.org/10.1145/3456887.3459697

11 T. H. Soe: Proc. Workshop on Automation Experience at the Workplace (2021) 1-5.

12 M. Leake, A. Davis, A. Truong, and M. Agrawala: ACM Trans. Graphics 36 (2017) 1. https://doi. org/10.1145/3072959.3073653

13 T. Hirai: J. Global Media Stud. (2018) 71.

14 Y. Perwej, M. K. Omer, O. E. Sheta, H. M. Harb, and M. S. Adrees: Int. J. Eng. Sci. Comput. (2019) 20193.

15 W. T. Sung and S. J. Hsiao: Sens. Mater. 32 (2020) 115. https://doi.org/10.18494/SAM.2020.2569

16 W. L. Hsu, W. T. Chen, H. H. Kuo, Y. C. Shiau, T. Y. Chern, S. C. Lai, and W. H. Fan: Sens. Mater. 32 (2020) 183. https://doi.org/10.18494/SAM.2020.2581

17 T. W. Chang, Y. S. Wu, S. Datta, and W. L. Mao: Sens. Mater. 32 (2020) 2425. https://doi.org/10.18494/ SAM.2020.2811

18 New Taipei City Police Department: Traffic Violation Reporting System. https://tvrs.ntpd.gov.tw/ (accessed May 2021).

19 Rclone: https://rclone.org/install/ (accessed June 2021).

20 Rsync for cloud storage: https://github.com/rclone/rclone (accessed June 2021). 


\section{About the Authors}

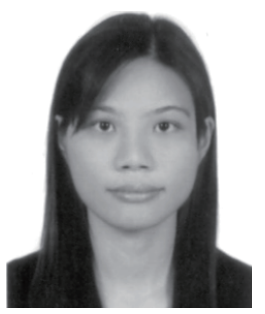

Wen-Chuan Wu received her B.S. degree from Tung-Hai University, Taiwan, in 2001 and her M.S. and Ph.D. degrees from National Chung-Cheng University, Taiwan, in 2003 and 2007, respectively. Currently, she is an assistant professor in the Department of Computer Science and Information Engineering, National Ilan University, Taiwan. Her research interests are image processing, data compression, interactive multimedia, information security, IoT, and STEAM education. (wenn@niu.edu.tw)

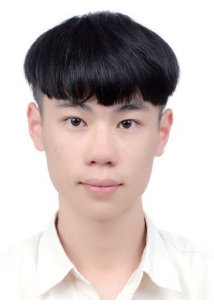

Yen-Lun Chen is currently a junior undergraduate of the Department of Computer Science and Information Engineering, Aletheia University, Taiwan. (allen.0917.5859@gmail.com)

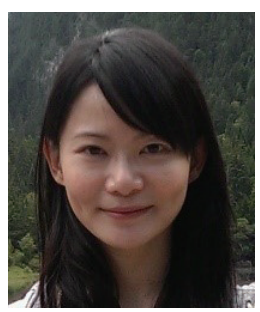

Pei-Yu Lin is an associate professor in the Department of Information Communication, Yuan Ze University, Taiwan. Her current research interests include image protection, data mining, and information security.

(pagelin3@gmail.com) 